\title{
Advances of exosome in the development of ovarian cancer and its diagnostic and therapeutic prospect
}

This article was published in the following Dove Press journal:

OncoTargets and Therapy

\author{
Jiayu Shen \\ Xiaoqing Zhu \\ Jing Fei \\ Pengyao Shi \\ Shuqian Yu \\ Jianwei Zhou
}

Department of Gynecology, The Second Affiliated Hospital of Zhejiang University School of Medicine, Hangzhou, People's Republic of China
Correspondence: Jianwei Zhou Department of Gynecology, The Second Affiliated Hospital of Zhejiang University School of Medicine, No 88, Jiefang Road, Shangcheng District, Hangzhou, Zhejiang 310002, People's Republic of China Email 2195045@zju.edu.cn

\begin{abstract}
Ovarian cancer is the leading cause of female gynecological cancer mortality. Most patients with ovarian cancer are diagnosed with advanced stage because of lack of early symptoms, physical signs, and sensitive tumor biomarkers. The standard treatment includes cytoreductive surgery and platinum-based chemotherapy (usually platinum combined with paclitaxel). Despite that postoperative adjuvant chemotherapy prolongs survival time, most patients go through relapse within 6-12 months after the treatment. Thus, elucidating the molecular mechanism in cancer development is essential to promote early diagnosis and novel treatments. The role of exosome has been highlighted in multiple research fields in recent years. Exosome has been described as nano-sized vesicle secreted by multiple mammalian cell types, carrying cargos like proteins, miRNAs, mRNAs, and lipids. It participates in the formation of tumor microenvironment and the development of tumorigenesis and drug resistance in ovarian cancer. Meanwhile, it may also play a pivotal role in diagnosis, efficacy evaluation, and prognosis. Besides, studies show that exosome and its processed products have promising value in ovarian cancer treatment. The aim of the current review is to describe the characteristics of exosome in ovarian cancer, especially focusing on its role in immune modulation and drug resistance, hoping to provide new information on its implications in cancer diagnosis and treatment.
\end{abstract}

Keywords: ovarian cancer, exosome, tumor development, immune regulation, drug resistance, diagnosis, treatment

\section{Introduction}

Ovarian cancer is one of the top three common malignancies and the deadliest disease in the female gynecological system. The most common tumor type is epithelial ovarian cancer (EOC), accounting for $\sim 90 \% .{ }^{1,2}$ Because of lack of representative symptoms and sensitive diagnostic approaches, more than $70 \%$ patients present with extra-pelvic lesions and are diagnosed with advanced stage (FIGO stage III or IV). ${ }^{3}$ Despite that novel therapies are emerging in recent years, such as antiangiogenesis and targeted therapy, little survival improvement has been achieved. At present, the standard therapy of ovarian cancer remains cytoreductive surgery and postoperative adjuvant chemotherapy with platinum-based compounds. According to the National Comprehensive Cancer Network guidelines, recommendations regarding adjuvant therapy include intravenous with or without intraperitoneal options, such as paclitaxel/ carboplatin regimen, docetaxel/carboplatin, doxorubicin/carboplatin, and cisplatin/ paclitaxel. ${ }^{4-6}$ All of regimens have different toxicity profiles. For example, the intravenous paclitaxel/carboplatin regimen is associated with sensory peripheral neuropathy. The intraperitoneal paclitaxel/cisplatin regain is associated with leukopenia, 
infection, fatigue, renal toxicity, abdominal discomfort, and neurotoxicity. ${ }^{7,8}$ In addition to the toxicities, drug resistance also remains as an obstacle in cancer treatment. Patients show response to chemotherapy initially, but many patients develop drug resistance in 6-12 months with the increasing use of cytotoxic agents. Despite that adjuvant chemotherapy does improve patients' outcome, the 5-year survival rate of advanced patients is still low $(<30 \%)$ due to late diagnosis and drug resistance. Contrarily, the 5-year survival rate of patients with early stage (FIGO stage I or II) can reach $90 \%$. ${ }^{3,9}$ Such outcomes highlight the urgency of implementing early diagnostic methods and treatment strategies for ovarian cancer with novel, more effective, and less toxic approaches.

Exosome has been widely investigated in recent years. It has been described as a nano-sized vesicle encapsulated with lipid bilayer. Exosome can be found in different body fluids, such as plasma, saliva, urine, latex, cerebrospinal fluid, and ascites. It carries various cargos such as proteins, nucleic acid, and lipids reflecting the cell of origin, and exerts as a vehicle to transport cargos to cells. Exosome plays a key role in cell-cell communication by releasing bioactive molecules, fusing with the membrane of recipient cell or combing with the receptors on cell surface. ${ }^{10-16}$ As one part of tumor microenvironment, exosome participates in cell proliferation, angiogenesis, metastasis, immune regulation, drug resistance, and pre-metastatic environment formation. ${ }^{10,16-19}$ Recent studies revealed a strong connection between exosome and ovarian cancer. Exosome may play an important role in the progression and drug resistance of ovarian cancer. Moreover, it is a promising biomarker for the diagnosis and novel therapeutic strategies in the ovarian cancer. The aim of the current review is to describe the characteristics of exosome in ovarian cancer, especially focusing on its role in immune modulation and drug resistance, hoping to provide new information on its implications in cancer diagnosis and treatment.

\section{The structure of exosome}

Exosome, first found in maturing mammalian reticulocytes in 1983, is a nano-vesicle ranging from 30 to $100 \mathrm{~nm}$ in diameter. ${ }^{20}$ Different from microvesicles (100 nm to $\left.1 \mu \mathrm{m}\right)$ separated from the membrane directly, exosome is mainly formed through the endosomal pathway. ${ }^{21-24}$ The biogenesis of exosome begins with inward invagination of the cell membrane, causing the incorporation of membrane proteins in the early endosome. As the early endosome matures and develops into the late endosome, it undergoes inward budding resulting in the formation of intraluminal vesicles. The late endosome containing intraluminal vesicles is also called as multivesicular body (MVB). When the MVB forms, it may go two different ways. Typically, it fuses with the lysosome and then its contents are degraded by the hydrolysis. Otherwise, instead of fusion with the lysosome, the MVB may fuse with the cell membrane, releasing its intraluminal vesicles to the extracellular environment. These vesicles are then described as exosomes (Figure 1). As mentioned already, exosome has been identified in many body fluids, such as blood, saliva, urine, ascites, breast milk, amniotic fluid, and etc. It can be secreted under both normal and pathological conditions and by a wide array of mammalian cell types, including lymphocytes, dendritic cells (DCs), macrophages, mast cells, stem cells, epithelial cells, endothelial cells, as well as cancer cells. ${ }^{25-31}$ Notably, cancer cells are said to produce exosomes in greater amounts than normal cells due to the possible factors (like stress, hypoxia, and cytokines) existing in the tumor microenvironment. ${ }^{32-34}$

Exosome is an informative vesicle rich in proteins, nucleic acid, and lipids. The molecule composition of the exosome depends in part on the type and state of the originating cell. Exosome includes multiple categories of proteins, such as proteins involved in antigen presentation (such as CD1, the major histocompatibility molecules [MHC] class I and II), tetraspanins (CD9, CD63, CD81, and CD82), proteins involved in structure construction (like tubulin, actin, and filament actin), cytoplasmic proteins (such as annexins, Rab proteins, flotillin, Alix, and TSG101), and signaling transduction-related proteins (like protein kinases and $\mathrm{G}$ proteins). Other common exosomal proteins include heat shock proteins (HSP) 70 and HSP90, as well as some proteins which exist in tumor-derived exosome (like Fas ligand [FasL]) in tumor-derived exosome. ${ }^{31,35-46}$ In addition to proteins, exosome has been reported to contain nucleic acid such as DNA, mRNA, miRNA, and non-coding RNA, which plays a role in angiogenesis, exocytosis, and tumorigenesis by modulating gene expression and protein translation. ${ }^{47-51}$ Exosome also contains lipids that may be related to maintain the morphology of exosome and exert as a signaling molecule. ${ }^{52}$ Recent information from different cell types reveals that exosome contains 41,860 proteins, 1,116 lipids, 4,946 mRNA, and 2,838 miRNA. Since exosome carries multiple functional molecules, its role in disease development draws more attention.

\section{Exosome's role in ovarian cancer}

Exosome participates in transportation, cell-cell communication, signal transduction, antigen presentation, and immune regulation by releasing bioactive molecules, fusing with 


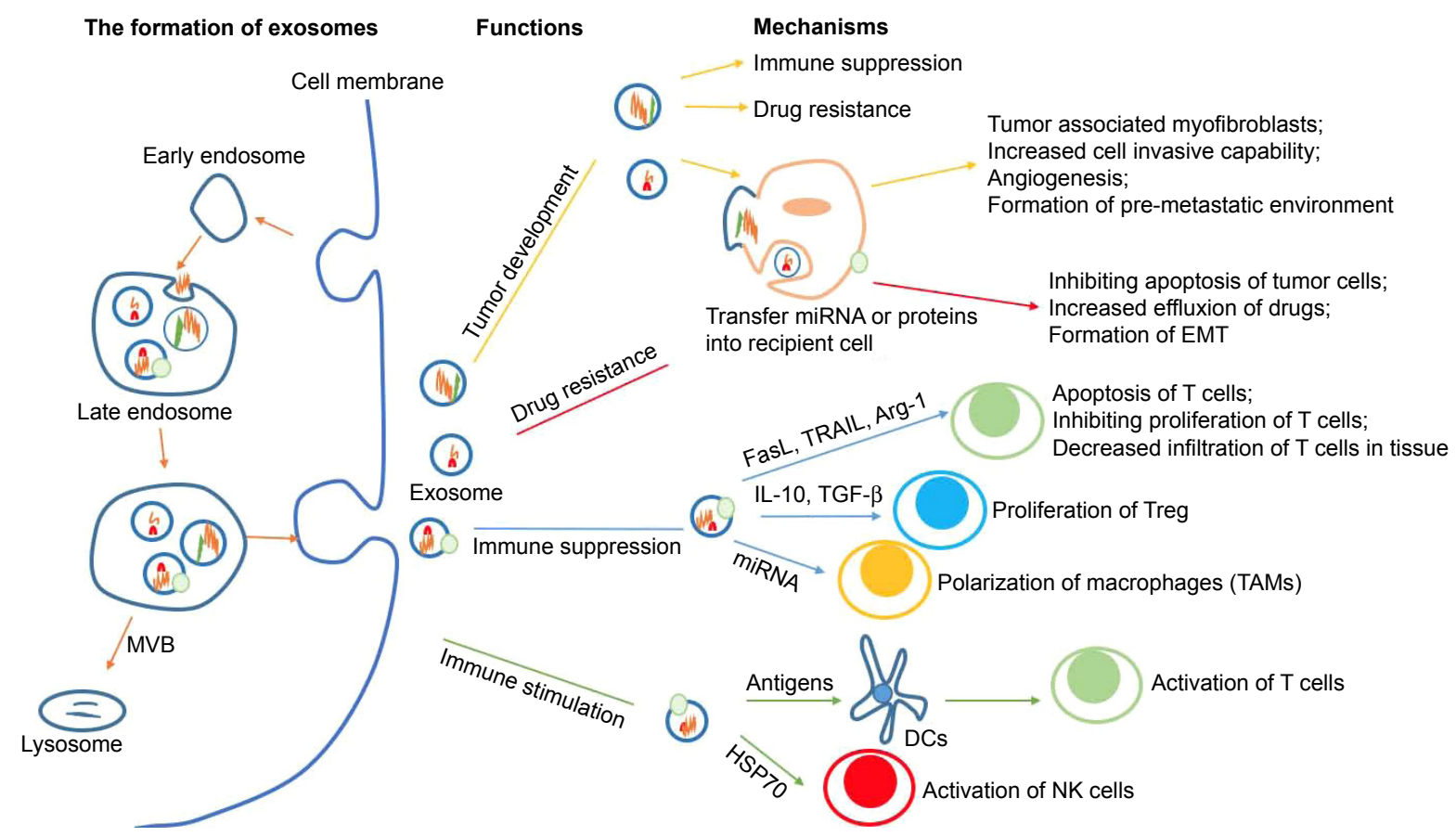

Figure I The formation process of exosomes and their roles in ovarian cancer. ${ }^{22-24}$

Notes: The biogenesis of exosome begins with inward invagination of the cell membrane. As the early endosome develops into the late endosome, it undergoes inward budding, leading to the formation of MVB. MVB then fuses with the cell membrane, releasing exosomes to the extracellular environment. Ovarian cancer-derived exosomes may be involved in tumor development, drug resistance, and immune regulation via transferring different molecules like proteins and miRNAs.

Abbreviations: Arg-I, arginase-I; DC, dendritic cell; EMT, epithelial-mesenchymal transition; FasL, Fas ligand; HSP70, heat shock protein 70; IL-I0, interleukin-I0; TRAIL, TNF related apoptosis-inducing ligand; MVB, multivesicular body; NK, natural killer; TAM, tumor-associated macrophage; TGF- $\beta$, transforming growth factor- $\beta$; Treg, regulatory T cells.

the membrane of recipient cell, or combing with the receptors on cell surface, which is related to both biological and pathological procession. Recently, the relationship between exosome and ovarian cancer has attracted much more attention. Like other exosomes derived from normal cells, exosomes from ovarian cancer also contain lots of bioactive molecules that may be dominants in tumor progression, such as immune regulation related molecules (MHCI and HSP), miRNA involved in tumor invasion (miR-200 and miR-222-3p), and drug resistance-related molecules (miR-21, annexin A3). The characteristics of exosome in ovarian cancer mainly consists of the following five aspects.

\section{Exosome in modulating tumor development}

Tumor-derived exosome is one part of tumor microenvironment, involving in promoting cell proliferation, angiogenesis, metastasis, and the formation of pre-metastatic environment. Cho et al found that the adipose tissue-derived mesenchymal stem cells (ADSCs) treated with ovarian cancer-derived exosomes could be induced to exhibit the typical characteristics of tumor-associated myofibroblasts with increased expression of $\alpha$-smooth muscle actin and also increased secretion of stromal derived factor- 1 and transforming growth factor (TGF)- $\beta$. Meanwhile, the upregulated expression of TGF- $\beta$ receptor isotypes (TGF- $\beta$ RI and II) suggested that the generation of myofibroblasts from ADSCs can be related to the activation of TGF- $\beta$ receptor mediated downstream signaling pathways. Further study revealed that exosomes from different originating cells activated different signaling pathways. Exosomes from SKOV3 increased the expression of phosphorylated SMAD2 to activate SMAD-dependent pathway, while OVCAR3-derived exosomes increased the phosphorylation form of AKT to activate the representative SMAD-independent pathway (PI3K/AKT pathway). ${ }^{53}$ All of the aforementioned results clearly indicate that tumor-derived exosomes can induce the tumor-associated myofibroblastic phenotype by creating appropriate microenvironment and thereby contribute to the progression and malignancy of tumor cells. Ovarian cancer is characteristic of abdominal metastasis, such as omentum and peritoneum. Study conducted by Nakamura et al indicated that exosome-mediated transfer played a functional role in abdominal metastasis. Human peritoneal mesothelial cells (HPMCs) isolated from normal omentum were treated with exosomes derived from different ovarian cancer cell lines separately. Results showed that exosome-treated HPMCs changed in cellular morphology to spindle phenotype and the invasion capability was significantly promoted. Furthermore, they revealed that exosomes transferred CD44 to HPMCs, which could upregulate the secretion of matrix metalloproteinase- 9 and increase the 
clearance of HPMCs, resulting in cell invasion promotion. ${ }^{54}$ Additionally, miRNA is reported to play a crucial role in tumor progression by modulating gene expression. For instance, EOC-derived exosomes can transfer miR-222-3p to macrophages to induce the polarization of tumor-associated macrophage (TAM)-like phenotype with SOCS3/STAT3 pathway involvement, resulting in tumor progression and metastasis by promoting cell proliferation, angiogenesis, and extracellular matrix degradation. ${ }^{55,56}$ Kanlikilicer et al found that the expression of miR-1246 was significantly increased in ovarian cancer exosomes and miR-1246 could inhibit RB tumor suppressor protein and regulate Caveolin-1 and platelet-derived growth factor receptor $\beta$ precursor signaling to mediate tumorigenesis. ${ }^{57}$ Exosome miR-101 was reported to repress ovarian cancer cell migration and invasion by inhibiting brain-derived neurotrophic factor. $\mathrm{Xu}$ et al's study revealed that the expression level of miR-101 was significantly decreased in ovarian cancer exosomes, indicating the reduction of miR-101 is related to enhanced cancer cell migration and invasion. ${ }^{58}$ Taken together, tumor-derived exosomes can alter the invasive and metastatic capacity of ovarian cancer cells by transferring informative molecules. Additionally, exosome can also promote tumor progression via inhibiting immune system or enhancing drug resistance, which will be discussed in the following text. Anyway, what has been found so far is only the tip of iceberg. Further studies are needed to fully elucidate the characteristics of exosome in ovarian cancer development.

\section{Exosome in immune regulation}

Throughout the tumor microenvironment, multiple immune cells, including $\mathrm{T}$ and B lymphocytes, natural killer (NK) cells, and macrophages, exert functions depending on the tissue composition and stimuli. Immune responses can be regulated by different components, such as proteins and RNAs. As one focal member in the tumor microenvironment, exosomes carry informative cargos and their role in modulating immune system has been highlighted. Tumorderived exosomes have been shown to inhibit the immune system via different mechanisms. For example, exosomes can induce the apoptosis of effective T lymphocytes through Fas/FasL pathway or downregulate the activity of NK cells via NKG2D ligands. ${ }^{59-61}$ Specifically, in ovarian cancer, exosomes could promote anti-tumor response via enhancing the apoptosis of peripheral lymphocytes and DCs through Fas/FasL pathway or by secreting exosomal TRAIL, which is an apoptosis-induced signal molecule. ${ }^{62}$ It is also said that exosomes can collectively induce T-cell apoptosis by increasing the expression of FasL on the exosome surface and suppressing CD3-zeta on T cells. ${ }^{63,64}$ Besides, ex vivo experiment revealed that ascites-associated exosomes with high expression of arginase-1, which was associated negatively with intratumoral T-cell infiltration and CD3-zeta expression, could act as an immunosuppressor by inhibiting the proliferation of $\mathrm{CD}^{+}$and $\mathrm{CD}^{+} \mathrm{T}$ cells and decreasing the number of T-cell receptors. ${ }^{65}$ Tumor-derived exosomes have also been reported to enhance immune suppression by promoting the differentiation of inhibitory immune cells, including myeloid-derived suppressor cells (MDSC), TAMs, and regulatory T cells (Treg). MDSC can negatively modulate antigen processing and presentation and increase the secretion of immunosuppressive factors. Exosomes can induce the differentiation of MDSC and promote the infiltration of MDSC in tumor tissue to inhibit immune response. ${ }^{66,67}$ Speaking of Treg, Szajinik et al found that plasma exosomes from patients with ovarian cancer could activate the conversion of $\mathrm{CD}^{+} \mathrm{CD} 25^{\text {neg }} \mathrm{T}$ cells into $\mathrm{CD} 4^{+} \mathrm{CD} 25^{\text {high }} \mathrm{FOXP} 3^{+}$ Treg, which was responsible for inhibitory functions of $\mathrm{CD} 4^{+} \mathrm{CD} 25^{\text {neg }} \mathrm{T}$ cells and $\mathrm{CD}^{+} \mathrm{T}$ cells. It appeared that exosomes not only induced Treg, but also promoted Treg proliferation and enhanced the effects of immunosuppression via mechanisms involving interleukin (IL)-10 and TGF- $\beta .^{68}$ As already mentioned, exosomes can transfer miR-222-3p to macrophages to induce the polarization of TAM-like phenotype, which plays an important role in immune suppression. Additionally, exosomes can facilitate the formation of immunosuppressive microenvironment through induction of cytokines. Ascites-derived exosomes can be uptaken by the monocytes, then activate the Toll-like receptor (TLR)dependent signaling pathway, and induce the increased secretion of cytokines like IL-1 $\beta$, tumor necrosis factor- $\alpha$, and IL-6, resulting in immune suppression. ${ }^{69}$

Interestingly, some tumor-derived exosomes are found to exert a positive regulation of immune response to prevent tumor progression. The tumor-associated antigens carried by exosomes can be recognized by antigen presenting cells to stimulate immune cells, resulting anti-tumor response. ${ }^{70,71}$ Several studies found that tumor antigens carried by exosomes could be recognized by DCs and then induced the cytotoxic activity of effective $\mathrm{T}$ lymphocytes, resulting in inhibition of tumor growth. ${ }^{72,73}$ Exosomes derived from pancreatic cancer or liver carcinoma contain HSP70, which can activate NK cells to promote apoptosis of tumor cells via granzyme B and perforin. ${ }^{74,75}$ In vivo experiments showed that HSP-contained melanoma exosomes could inhibit tumor growth and metastasis via activating NK cells in mouse 
model. ${ }^{76}$ In ovarian cancer, Li et al found that ascites-derived exosomes carrying tumor-specific antigens MHCI, HSP70, HSP90, Her2/Neu, and Mart1, which could be presented by DCs, could induce the activation of cytotoxic T cells, resulting in immune response. ${ }^{77}$ Anyhow, few researches mentioned exosomes' role in immune promotion in ovarian cancer. Taken the immune stimulating role of exosomes in other tumor types into consideration, exosomes from ovarian cancer may also enhance the immune response by activating the NK cells or cytotoxic T cells, which may become another hot issue in research field.

Nevertheless, whether exosomes play a suppressor or an activator role in immune system of ovarian cancer remains unknown. Lots of factors can affect the immune system, including tumor types, immune cells, cytokines, or host conditions. Further studies are needed to elicit the mechanisms.

\section{Exosome in drug resistance}

Drug resistance remains a major obstacle in cancer treatment. Elucidating the molecular mechanism in cancer development and drug resistance is essential to promote novel treatment strategies. In recent years, the role of exosomes in drug resistance has been identified in many tumors, such as ovarian cancer, breast cancer, prostate cancer, and gastric cancer. ${ }^{78-80}$ For instance, exosome could induce increased docetaxel resistance in prostate cancer cells by transferring multidrug resistance protein 1 (MDR1). ${ }^{78}$ In gastric cancer, exosomes from mesenchymal stem cells could activate calcium/calmodulin-dependent protein kinases (CaM-Ks) and Raf/MEK/ERK kinase cascade and increase the expression of multidrug resistance-associated protein (MRP), MDR, and lung resistance protein, resulting in inhibition of 5 -Fu induced apoptosis. ${ }^{79}$ In ovarian cancer, the phenomenon of the negative correlation between intracellular drug concentration and exosome secretion suggests that exosome plays a pivotal role in drug resistance. So far, the identified possible mechanisms of exosome-associated resistance mainly include the following three aspects: 1) transfer drug resistance-related miRNAs (ie, miR-21, miR-21-3p, miR-433) to recipient cells and then modulate gene expression and induce resistance, ${ }^{81-84}$ 2) transfer drug exporting proteins (ie, annexin A3, P-glycoprotein, ATP7A, ATP7B) to decrease intracellular concentration; ${ }^{85-88}$ and 3 ) induce the characteristics of epithelial-mesenchymal transition (EMT) cell morphology. ${ }^{89}$ However, it is worth noticing that exosome's complexity means that other mechanisms may exist and additional studies are warranted.
1) Exosomal miRNAs in drug resistance: miRNA is small non-coding RNA with 21 nucleotides in length and can modulate gene expression. Interestingly, it has been reported that exosomes can transfer miRNAs between cells, contributing to drug resistance. A few studies uncover new insight into the effects of exosomal miRNAs in drug resistance in ovarian cancer. Au Yeung et al found that exosomes isolated from cancer-associated adipocytes (CAAs) and fibroblasts (CAFs) expressed significantly higher level of miR-21. Further studies showed that the apoptosis was suppressed and chemoresistance was induced in ovarian cancer cells treated with exosomes from CAAs or CAFs via suppressing apoptotic protease activation factor 1 . These results suggested that exosomal miR-21 could alter the malignant phenotype and drug response of ovarian cancer cells. ${ }^{82}$ Study conducted by Pink et al implicated that exosomal miR-21-3p (the star strand of miR-21) could increase ovarian cancer cells' resistance to cisplatin by suppressing the expression of neuron navigator $3 .^{83}$ Except for miR-21 and its star strand miR-21-3p, exosomal miR-433 was also reported to promote resistance to paclitaxel via affecting the tumor microenvironment by diminishing the proliferation of neighboring cells with the induction of cellular senescence, which may be related to the downregulation of cyclin-dependent kinase $6 .^{84}$ Though just a small number of studies reported that exosomal miRNAs played a role in drug resistance in ovarian cancer, other exosomal miRNAs, except for the miRNAs as already mentioned, may also be enlisted in drug resistance, which requires further studies to elucidate.

2) Exosomal proteins in drug resistance: Except for miRNAs, exosome also carries a wide range of proteins. A few studies have focused on the relationship between exosomal proteins and drug resistance in ovarian cancer, implicating that exosomes can transfer proteins to enhance resistance by inhibiting apoptosis or promoting drug effluxion.

Annexin A3: Annexin A3 belongs to the phospholipid binding proteins family, participating in membrane fusion, exocytosis, ion channels modulation, and signaling transduction. ${ }^{90}$ Yin et al found that annexin A3 was upregulated in platinum-resistant ovarian cancer cell lines and it selectively conferred resistance through decreasing intracellular concentration of platinum compounds and inhibiting cancer cell apoptosis. Further studies showed that upregulation of annexin A3 in cancer cells led to the formation of MVB-like vesicles, which could release 
exosomes through exocytosis. Additional immunoblotting and immunoelectron microscopy revealed that annexin A3 could be detected in exosomes, suggesting that exosomes may serve as vehicles to transfer annexin A3 between cells to induce drug resistance. ${ }^{85,86}$

$\mathrm{P}$-glycoprotein (P-gp): It is a $170 \mathrm{kDa}$ protein coded by $M D R-1$ gene and is one of the most well-studied mechanism of drug resistance in many tumor types. P-gp plays a pivotal role in maintaining appropriate intracellular drug concentration by promoting effluxion. The overproduction of P-gp will certainly lead to less effective response by over exporting drug. ${ }^{91,92}$ Recent studies showed that the expression level of P-gp in microvesicles from platinum-resistance A2780 cells was significantly higher compared with the chemosensitive wild-type (WT) parental line A2780/WT. Further studies demonstrated that microvesicles derived from resistant A2780 cells could transfer functional P-gp to A2780/WT cells and induce more than 5-fold higher resistance to adriamycin and paclitaxel in A2780/WT. ${ }^{87}$ Considering that exosome and microvesicles both belong to extracellular vesicles involved in cell-cell communication and exosomal P-gp has been demonstrated to induce resistance in breast cancer, exosome may also promote resistance in ovarian cancer by transferring P-gp. ${ }^{93}$ Nevertheless, further experiments are needed to identify the role of exosomal $\mathrm{P}$-gp in ovarian cancer resistance.

DNA methyltransferase 1 (DNMT1): DNMT1 plays an important role in the methylation maintenance during DNA replication and repair. Aberrant DNA methylation is involved in tumor resistance by modulating gene expression. ${ }^{94}$ Intriguingly, compared with exosomes from endometrial stromal cell line, a significant higher expression of DNMT1 was observed in exosomes from ovarian cancer cell line SKOV3. Cisplatin was used to treat SKOV3 with or without exposure to exosomes derived from SKOV3, and results showed that the apoptosis of the SKOV3 unexposed to exosomes was significantly higher. Additional studies revealed that treatment with GW4869 (exosome secretion inhibitor) abolished the resistance. ${ }^{95}$ All of the results suggested that exosomes could promote drug resistance by transferring DNMT1.

Others: In addition to annexin A3, P-gp, and DNMT1, drug export transporters proteins also have been reported in drug resistance. By comparing the secretion level in cisplatin-resistant ovarian cancer cell line 2008/ C13*5.25 and sensitive cells, exosomes were detected in larger quantity in 2008/C13*5.25 accompanied by higher expression of cisplatin export transporters MRP2, ATPase copper transporting ATP7A and ATP7B. Besides, exosomes released from $2008 / \mathrm{C} 13 * 5.25$ previously treated with cisplatin contained 2.6-fold more cisplatin than those from sensitive cells. ${ }^{88}$ These data revealed that exosome induced drug resistance by exporting putative transporters loaded with platinum compounds.

3) Exosome induced EMT in drug resistance: EMT is considered to be one mechanism of drug resistance for escaping from the deleterious effects of cytotoxic agents. ${ }^{96-98}$ Crow et al treated A2780 with exosomes derived from resistant A2780 derivatives CP70, C30, and C200 cells, which displayed a mesenchymal morphology. The results showed that the epithelial markers dystroglycan, E-cadherin, and epithelial cell adhesion molecule (EpCAM) were significantly downregulated, while the mesenchymal markers paladin, TWIST, and vimentin were upregulated, suggesting that exosome could induce EMT. ${ }^{89}$ Yew et al found that exposure to epimorphin induced reversal of EMT and led to an enhanced sensitivity to platinum in ovarian cancer cells. ${ }^{99}$ Taken together, exosome may induce the characteristics of EMT cell morphology to involve in resistant ovarian cancer.

\section{Exosome in diagnosis and prognosis prediction}

The main diagnostic approaches for ovarian cancer include biomarker CA125 and imaging. Anyway, CA125 is usually not elevated in the early stage and not every ovarian cancer patient exhibits increased level of serum CA125. Besides, other diseases with high level of CA125 may cause confusion, such as endometriosis, pelvic inflammation, and breast cancer. Therefore, it is of vital importance to find novel biomarkers that can be detected in early stage and present typical characteristics of ovarian cancer. Exosome is an informative vesicle that carries proteins and nucleic acids representative of its originating cell, suggesting its diagnostic and prognostic value. As a diagnostic tool, exosome has the following advantages. First of all, exosome is abundant in plasma and other body fluids, making its advantage overweigh invasive biopsies. Secondly, exosome is extremely stable and can be stored under various conditions (freezing, cold-storage, or thawing) for months and even years. The cargos exosome carries are tumor specific and correlates with tumor staging and prognosis. Compared with exosomes derived from benign ovarian lesions, exosomes from ovarian cancer contain significantly increased level of TGF $\beta 1$ and melanoma-associated antigen (MAGE) 3 and MAGE6, 
suggesting potential biomarkers for distinguishing malignancy from benignity. ${ }^{100} \mathrm{Li}$ et al found that the expression level of EOC representative biomarker Claudin 4 in serum exosomes from ovarian cancer was positively connected with tumor staging. ${ }^{101}$ Other markers, such as CD24 and EpCAM, are also exploited for diagnostic value in ovarian carcinoma. ${ }^{37}$ Chemoresistance-related proteins are highly expressed in exosomes originated from drug resistant cancer cells, such as annexin A3, MRP2, ATP7A, and ATP7B, indicating the potential value to predict the therapeutic response to chemotherapy in patients with ovarian cancer. . $^{85,86,88,102}$ Additionally, the use of miRNA profile in exosomes is also shown to be highly informative for early diagnosis in ovarian cancer. Previous studies showed that the expression of miR21, miR-141, miR-200a, miR-200b, miR-200c, miR-203, miR205, and miR-214 was significantly elevated in ovarian cancer exosomes. ${ }^{103}$ Anyhow, despite of the potential value of exosome's contents in diagnosis and prognosis, lots of problems remain to be dealt with. For example, the limitation to isolate pure exosomes could impact overall study of exosomes. The secretion of exosomes can be influenced by many factors, such as stress, hypoxia, tumor types, and growth patterns. For instance, hypoxia is a hallmark of tumor microenvironment that arises due to imbalance of the supply and consumption of oxygen by tumor cells, leading to a low $\mathrm{pH}$ condition where tumor cells can survive, while normal cells cannot. Studies showed that exposure of breast cancer cells to modest $(1 \%)$ and severe hypoxia $(0.1 \%)$ resulted in mean increases of $32.3 \% \pm 4.8 \%$ and $90.9 \% \pm 7.1 \%$ of exosomes, respectively. Meanwhile, it is also shown that hypoxic tumor-derived exosomes contain various pro-angiogenic factors, which allow them to promote angiogenesis and endothelial cell activation..$^{32,33,104}$ Other studies also showed that low extracellular $\mathrm{pH}$ induced an increased release of exosomes by human melanoma cells and counteracting the low $\mathrm{pH}$ markedly reduced the exosome release from tumor cells. ${ }^{105,106}$ All these data suggest that the specific condition of tumor microenvironment affects the amounts and cargos of exosomes. Therefore, further studies are still needed to confirm its practical application in diagnosis.

\section{Exosome in cancer treatment}

Exosomes in therapeutic field are continuously being investigated and developed. With the growing knowledge of exosomes' role in cancer, it is possible to reduce the secretion of tumor-associated exosomes, load drugs or miRNAs on exosomes, or develop exosome-based immunotherapy. Some studies have already proven exosomes to be a potential tool of treatment. In stage III/IV melanoma patients, a Phase I clinical trial was conducted to testify the feasibility and safety of autologous exosomes pulsed with MAGE3 peptides for immunotherapy. ${ }^{107}$ In breast cancer, ex vivo experiment showed that $\beta$-elemene could reverse chemoresistance of breast cancer cells via reducing resistance transmission by exosomes. ${ }^{108}$ As to ovarian cancer, $\mathrm{Hu}$ et al found that exosomes isolated from macrophages that were previously treated with tumor necrosis factor-like weak inducer of apoptosis (TWEAK) could inhibit the migration and invasion of EOC by upregulating the expression of miR-7 and then inhibiting the activation of EGFR/AKT/ERK1/2 pathway, indicating that exosomal miRNA-7 from TWEAK-stimulated macrophages may have promising therapeutic prospects. ${ }^{109,110}$ IRGD exosomes, which were constructed via transfecting plasmid with tumor targeting peptide IRGD to exosomes, were observed to selectively interact with ovarian cancer cell NuTu-19 through membrane fusion. IRGD exosomes loaded with adriamycin (Dox) were then intravenously injected into mice with ovarian cancer. The results showed that the tumor volume of IRGD exosomes-Dox group was significantly smaller than that of PBS group, exosomes group, Dox group, and blank-exosomesDox group, uncovering that the construction of multifunctional exosome-based drug carrier has good application value in anti-tumor therapy. ${ }^{111}$ Similarly, Mahmoodzadeh Hosseini et al testified the cytotoxic impact of TEX-SEB (tumor-derived exosomes anchored with staphylococcal enterotoxin B [SEB]) on ovarian cancer cell SKOV3. Results showed that the proliferation was significantly inhibited and the apoptosis rate was increased in SKOV3 treated with TEX-SEB, indicating that exosome reconstruction can be a potential therapeutic method for ovarian cancer. ${ }^{112}$ Additionally, exosomes can carry molecules involved in antigen presentation, such as MHCI, HSP, tumor-associated antigens (ie, ovarian cancer antigens MAGE, NY-ESO, P53, Muc-1), indicating its immunotherapeutic prospects. ${ }^{72,77,113}$ For example, Andre et al found that exosomes isolated from malignant ascites constituted a source of tumor antigens that could be transferred to DCs and then the DCs caused differentiation and expansion of tumor-specific cytotoxic T lymphocytes, leading to anti-tumor response. ${ }^{114}$ A Phase I/II clinical trial is pending by Navabi et al to test the feasibility and therapeutic value of ascites-derived exosomes combined with TLR3 agonist for immunotherapy of advanced ovarian cancer. ${ }^{115}$ All abovementioned studies implicate the therapeutic prospects of exosome and its processed productions for ovarian cancer. Anyhow, these therapies warrant further additional animal studies and large cohort clinical trials to confirm efficacy and safety. 


\section{Prospects}

Ovarian cancer threatens female gynecological health. Difficulties in early diagnosis, high metastatic rate, and drug resistance remain the major obstacles. One attractive area is the role of exosomes in ovarian cancer. As one member of tumor microenvironment, exosomes play a pivotal role in tumor development, drug resistance, immunosuppression. As informative carriers between cells, exosomes have a promising value in early diagnosis and prognosis assessment. Besides, the therapeutic value of exosome in cancer treatment is under investigation. New ideas, such as the elimination of specific exosome or inhibiting exosome secretion to slow down tumor progression or improve drug sensitivity, whether exosomes transfected with specific miRNAs can reverse drug resistance or not and whether exosomes can be vaccinated for immunotherapy or not, are awaiting further studies to verify.

\section{Acknowledgment}

This research was supported by the National Natural Science Foundation of China (grant number 81371881).

\section{Disclosure}

The authors report no conflicts of interest in this work.

\section{References}

1. Chan JK, Cheung MK, Husain A, et al. Patterns and progress in ovarian cancer over 14 years. Obstet Gynecol. 2006;108(3 Pt 1):521-528.

2. Jelovac D, Armstrong DK. Recent progress in the diagnosis and treatment of ovarian cancer. CA Cancer J Clin. 2011;61(3):183-203.

3. Siegel RL, Miller KD, Jemal A. Cancer statistics. CA Cancer J Clin. 2017; 67(1):7-30.

4. Cristea M, Han E, Salmon L, Morgan RJ. Practical considerations in ovarian cancer chemotherapy. Ther Adv Med Oncol. 2010;2(3): 175-187.

5. Vasey PA, Jayson GC, Gordon A, et al; Scottish Gynaecological Cancer Trials Group. Phase III randomized trial of docetaxel-carboplatin versus paclitaxel-carboplatin as first-line chemotherapy for ovarian carcinoma. J Natl Cancer Inst. 2004;96(22):1682-1691.

6. Pignata S, Scambia G, Ferrandina G, et al. Carboplatin plus paclitaxel versus carboplatin plus pegylated liposomal doxorubicin as first-line treatment for patients with ovarian cancer: the MITO-2 randomized phase III trial. J Clin Oncol. 2011;29(27):3628-3635.

7. Wright JD, Hou JY, Burke WM, et al. Utilization and toxicity of alternative delivery methods of adjuvant chemotherapy for ovarian cancer. Obstet Gynecol. 2016;127(6):985-991.

8. Wenzel LB, Huang HQ, Armstrong DK, Walker JL, Cella D; Gynecologic Oncology Group. Health-related quality of life during and after intraperitoneal versus intravenous chemotherapy for optimally debulked ovarian cancer: a Gynecologic Oncology Group Study. J Clin Oncol. 2007;25(4):437-443.

9. Bristow RE, Chang J, Ziogas A, Campos B, Chavez LR, AntonCulver H. Impact of National Cancer Institute Comprehensive Cancer Centers on ovarian cancer treatment and survival. J Am Coll Surg. 2015; 220(5):940-950.

10. Ge R, Tan E, Sharghi-Namini S, Asada HH. Exosomes in cancer microenvironment and beyond: have we overlooked these extracellular messengers? Cancer Microenviron. 2012;5(3):323-332.
11. Koga K, Matsumoto K, Akiyoshi T, et al. Purification, characterization and biological significance of tumor-derived exosomes. Anticancer Res. 2005;25(6A):3703-3707.

12. Keller S, Sanderson MP, Stoeck A, Altevogt P. Exosomes: from biogenesis and secretion to biological function. Immunol Lett. 2006;107(2): 102-108.

13. van Niel G, Porto-Carreiro I, Simoes S, Raposo G. Exosomes: a common pathway for a specialized function. J Biochem. 2006;140(1):13-21.

14. Simons M, Raposo G. Exosomes - vesicular carriers for intercellular communication. Curr Opin Cell Biol. 2009;21(4):575-581.

15. Masyuk AI, Masyuk TV, Larusso NF. Exosomes in the pathogenesis, diagnostics, and therapeutics of liver diseases. J Hepatol. 2013;59(3): 621-625.

16. Beach A, Zhang HG, Ratajczak MZ, Kakar SS. Exosomes: an overview of biogenesis, composition and role in ovarian cancer. J Ovarian Res. 2014;7:14.

17. Rak J, Yu JL, Klement G, Kerbel RS. Oncogenes and angiogenesis: signaling three-dimensional tumor growth. J Investig Dermatol Symp Proc. 2000;5(1):24-33.

18. Valenti R, Huber V, Iero M, Filipazzi P, Parmiani G, Rivoltini L. Tumorreleased microvesicles as vehicles of immunosuppression. Cancer Res. 2007;67(7):2912-2915.

19. Psaila B, Lyden D. The metastatic niche: adapting the foreign soil. Nat Rev Cancer. 2009;9(4):285-293.

20. Pan BT, Johnstone RM. Fate of the transferrin receptor during maturation of sheep reticulocytes in vitro: selective externalization of the receptor. Cell. 1983;33(3):967-978.

21. Daveloose D, Wolf C, Jeanguillaume C, Leterrier F, Fabre G, Bereziat G. Inhibitory effect of microvesicles collected from stored blood on platelet aggregation. Thromb Res. 1981;22(1-2):195-201.

22. Théry C, Zitvogel L, Amigorena S. Exosomes: composition, biogenesis and function. Nat Rev Immunol. 2002;2(8):569-579.

23. Stoorvogel W, Kleijmeer MJ, Geuze HJ, Raposo G. The biogenesis and functions of exosomes. Traffic. 2002;3(5):321-330.

24. Bobrie A, Colombo M, Raposo G, Théry C. Exosomes secretion: molecular mechanisms and roles in immune response. Traffic. 2011;12(12): $1659-1668$

25. Raposo G, Nijman HW, Stoorvogel W, et al. B lymphocytes secrete antigen-presenting vesicles. $J$ Exp Med. 1996;183(3):1161-1172.

26. Blanchard N, Lankar D, Faure F, et al. TCR activation of human T cells induces the production of exosomes bearing the TCR/CD3/zeta complex. J Immunol. 2002;168(7):3235-3241.

27. Théry C, Regnault A, Garin J, et al. Molecular characterization of dendritic cell-derived exosomes. Selective accumulation of the heat shock protein hsc73. J Cell Biol. 1999;147(3):599-610.

28. Raposo G, Tenza D, Mecheri S, Peronet R, Bonnerot C, Desaymard C. Accumulation of major histocompatibility complex class II molecules in mast cell secretory granules and their release upon degranulation. Mol Biol Cell. 1997;8(12):2631-2645.

29. Lai RC, Arslan F, Lee MM, et al. Exosome secreted by MSC reduces myocardial ischemia/reperfusion injury. Stem Cell Res. 2010;4(3): 214-222.

30. Van Niel G, Mallegol J, Bevilacqua C, et al. Intestinal epithelial exosomes carry MHC class II/peptides able to inform the immune system in mice. Gut. 2003;52(12):1690-1697.

31. Henderson MC, Azorsa DO. The genomic and proteomic content of cancer cell-derived exosomes. Front Oncol. 2012;2:38.

32. Park JE, Tan HS, Datta A, et al. Hypoxic tumor cell modulates its microenvironment to enhance angiogenic and metastatic potential by secretion of proteins and exosomes. Mol Cell Proteomics. 2010;9(6): 1085-1099.

33. King HW, Michael MZ, Gleadle JM. Hypoxic enhancement of exosome release by breast cancer cells. BMC Cancer. 2012;12:421.

34. Hedlund M, Nagaeva O, Kargl D, Baranov V, Mincheva-Nilsson L. Thermal- and oxidative stress causes enhanced release of NKG2D ligand-bearing immunosuppressive exosomes in leukemia/lymphoma $\mathrm{T}$ and B cells. PLoS One. 2011;6(2):e16899. 
35. Logozzi M, De Milito A, Lugini L, et al. High levels of exosomes expressing CD63 and caveolin-1 in plasma of melanoma patients. PLoS One. 2009;4(4):e5219.

36. Almqvist N, Lönnqvist A, Hultkrantz S, Rask C, Telemo E. Serumderived exosomes from antigen-fed mice prevent allergic sensitization in a model of allergic asthma. Immunology. 2008;125(1):21-27.

37. Runz S, Keller S, Rupp C, et al. Malignant ascites-derived exosomes of ovarian carcinoma patients contain CD24 and EpCAM. Gynecol Oncol. 2007;107(3):563-571.

38. Saman S, Kim W, Raya M, et al. Exosome-associated tau is secreted in tauopathy models and is selectively phosphorylated in cerebrospinal fluid in early Alzheimer disease. J Biol Chem. 2012;287(6):3842-3849.

39. Hegmans JP, Bard MP, Hemmes A, et al. Proteomic analysis of exosomes secreted by human mesothelioma cells. Am J Pathol. 2004;164(5): 1807-1815.

40. Simpson RJ, Jensen SS, Lim JW. Proteomic profiling of exosomes: current perspectives. Proteomics. 2008;8(19):4083-4099.

41. Simpson RJ, Lim JW, Moritz RL, Mathivanan S. Exosomes: proteomic insights and diagnostic potential. Expert Rev Proteomics. 2009;6(3): 267-283.

42. Zöller M. Tetraspanins: push and pull in suppressing and promoting metastasis. Nat Rev Cancer. 2009;9(1):40-55.

43. Keller S, König AK, Marmé F, et al. Systemic presence and tumorgrowth promoting effect of ovarian carcinoma released exosomes. Cancer Lett. 2009;278(1):73-81.

44. Pfeffer SR. Two Rabs for exosomes release. Nat Cell Biol. 2010;12(1): 3-4.

45. McCready J, Sims JD, Chan D, Jay DG. Secretion of extracellular hsp90alpha via exosomes increases cancer cell motility: a role for plasminogen activation. BMC Cancer. 2010;10:294.

46. Taylor DD, Zacharias W, Gercel-Taylor C. Exosome isolation for proteomic analyses and RNA profiling. Methods Mol Biol. 2011;728: 235-246.

47. Bryant RJ, Pawlowski T, Catto JW, et al. Changes in circulating microRNA levels associated with prostate cancer. Br J Cancer. 2012; 106(4):768-774.

48. Taft RJ, Pang KC, Mercer TR, Dinger M, Mattick JS. Non-coding RNAs: regulators of disease. J Pathol. 2010;220(2):126-139.

49. Hannafon BN, Ding WQ. Intercellular communication by exosomederived microRNAs in cancer. Int J Mol Sci. 2013;14(7):14240-14269.

50. Valadi H, Ekström K, Bossios A, Sjöstrand M, Lee JJ, Lötvall JO. Exosome-mediated transfer of mRNAs and microRNAs is a novel mechanism of genetic exchange between cells. Nat Cell Biol. 2007;9(6) 654-659.

51. Thakur BK, Zhang H, Becker A, et al. Double-stranded DNA in exosomes: a novel biomarker in cancer detection. Cell Res. 2014;24(6): 766-769

52. Théry C, Amigorena S, Raposo G, Clayton A. Isolation and characterization of exosomes from cell culture supernatants and biological fluids. Curr Protoc Cell Biol. 2006; Chapter 3:Unit 3.22.

53. Cho JA, Park H, Lim EH, et al. Exosomes from ovarian cancer cells induce adipose tissue-derived mesenchymal stem cells to acquire the physical and functional characteristics of tumor-supporting myofibroblasts. Gynecol Oncol. 2011;123(2):379-386.

54. Nakamura K, Sawada K, Kinose Y, et al. Abstract 5060: Exosome transfer from ovarian cancer cells to mesothelial cells promotes cell invasion by up regulating MMP-9 secretion and increasing clearance of mesothelial cells. Cancer Res. 2015;75(Suppl 15):5060.

55. Wang X, Zhao X, Wang K, Wu L, Duan T. Interaction of monocytes/ macrophages with ovarian cancer cells promotes angiogenesis in vitro. Cancer Sci. 2013;104(4):516-523.

56. Ying X, Wu Q, Wu X, et al. Epithelial ovarian cancer-secreted exosomal miR-222-3p induces polarization of tumor-associated macrophages. Oncotarget. 2016;7(28):43076-43087.

57. Kanlikilicer P, Bayraktar R, Rashed M, et al. Abstract 1988: Exosomemediated ovarian cancer tumorigenesis mediated by miR1246/Rb/Cav1 axis. Cancer Res. 2017;77(Suppl 13):1988.
58. Xu Y, Xu L, Zheng J, Geng L, Zhao S. MiR-101 inhibits ovarian carcinogenesis by repressing the expression of brain-derived neurotrophic factor. FEBS Open Bio. 2017;7(9):1258-1266.

59. Andreola G, Rivoltini L, Castelli C, et al. Induction of lymphocyte apoptosis by tumor cell secretion of FasL-bearing microvesicles. J Exp Med. 2002;195(10):1303-1316.

60. Lundholm M, Schröder M, Nagaeva O, et al. Prostate tumor-derived exosomes down-regulate NKG2D expression on natural killer cells and CD8+ T cells: mechanism of immune evasion. PLoS One. 2014; 9(9):e108925.

61. Clayton A, Mitchell JP, Court J, Linnane S, Mason MD, Tabi Z. Human tumor-derived exosomes down-modulate NKG2D expression. J Immunol. 2008;180(11):7249-7258.

62. Peng P, Yan Y, Keng S. Exosomes in the ascites of ovarian cancer patients: origin and effects on anti-tumor immunity. Oncol Rep. 2011; 25(3):749-762.

63. Abrahams VM, Straszewski SL, Kamsteeg M, et al. Epithelial ovarian cancer cells secrete functional Fas ligand. Cancer Res. 2003;63(17): 5573-5581.

64. Morelli AE. The immune regulatory effect of apoptotic cells and exosomes on dendritic cells: its impact on transplantation. Am J Transplant. 2006;6(2):254-261.

65. Czystowska M, Szajnik M, Ramji K, Gruca S, Stefanowicz A, Nowis D. Abstract 3975: Ovarian cancer cells release arginase-1-containing exosomes to suppress antitumor immune response. Cancer Res. 2017 ; 77(Suppl 13):3975.

66. Xiang X, Poliakov A, Liu C, et al. Induction of myeloid-derived suppressor cells by tumor exosomes. Int J Cancer. 2009;124(11):2621-2633.

67. Liu Y, Xiang X, Zhuang X, et al. Contribution of MyD88 to the tumor exosome-mediated induction of myeloid derived suppressor cells. Am J Pathol. 2010;176(5):2490-2499.

68. Szajnik M, Czystowska M, Szczepanski MJ, Mandapathil M, Whiteside TL. Tumor-derived microvesicles induce, expand and upregulate biological activities of human regulatory T cells (Treg). PLoS One. 2010;5(7):e11469.

69. Bretz NP, Ridinger J, Rupp AK, et al. Body fluid exosomes promote secretion of inflammatory cytokines in monocytic cells via Toll-like receptor signaling. J Biol Chem. 2013;288(51):36691-36702.

70. Whiteside TL. Immune modulation of T-cell and NK (natural killer) cell activities by TEXs (tumor-derived exosomes). Biochem Soc Trans. 2013;41(1):245-251.

71. Bobrie A, Théry C. Exosomes and communication between tumours and the immune system: are all exosomes equal? Biochem Soc Trans. 2013;41(1):263-267.

72. Wolfers J, Lozier A, Raposo G, et al. Tumor-derived exosomes are a source of shared tumor rejection antigens for CTL cross-priming. Nat Med. 2001;7(3):297-303.

73. Zeelenberg IS, Ostrowski M, Krumeich S, et al. Targeting tumor antigens to secreted membrane vesicles in vivo induces efficient antitumor immune responses. Cancer Res. 2008;68(4):1228-1235.

74. Gastpar R, Gehrmann M, Bausero MA, et al. Heat shock protein 70 surface-positive tumor exosomes stimulate migratory and cytolytic activity of natural killer cells. Cancer Res. 2005;65(12):5238-5247.

75. Lv LH, Wan YL, Lin Y, et al. Anticancer drugs cause release of exosomes with heat shock proteins from human hepatocellular carcinoma cells that elicit effective natural killer cell antitumor responses in vitro. J Biol Chem. 2012;287(19):15874-15885.

76. Elsner L, Muppala V, Gehrmann M, et al. The heat shock protein HSP70 promotes mouse NK cell activity against tumors that express inducible NKG2D ligands. J Immunol. 2007;179(8):5523-5533.

77. Li QL, Bu N, Yu YC, Hua W, Xin XY. Exvivo experiments of human ovarian cancer ascites-derived exosomes presented by dendritic cell derived from umbilical cord blood for immunotherapy treatment. Clin Med Oncol. 2008;2(2):461-467.

78. Corcoran C, Rani S, O'Brien K, et al. Docetaxel-resistance in prostate cancer: evaluating associated phenotypic changes and potential for resistance transfer via exosomes. PLoS One. 2012;7(12):e50999. 
79. Ji R, Zhang B, Zhang X, et al. Exosomes derived from human mesenchymal stem cells confer drug resistance in gastric cancer. Cell Cycle. 2015;14(15):2473-2483.

80. Wei Y, Lai X, Yu S, et al. Exosomal miR-221/222 enhances tamoxifen resistance in recipient ER-positive breast cancer cells. Breast Cancer Res Treat. 2014;147(2):423-431.

81. O’Brien K, Rani S, Corcoran C, et al. Exosomes from triple-negative breast cancer cells can transfer phenotypic traits representing their cells of origin to secondary cells. Eur J Cancer. 2013;49(8):1845-1859.

82. Au Yeung CL, Co NN, Tsuruga T, et al. Exosomal transfer of stromaderived miR21 confers paclitaxel resistance in ovarian cancer cells through targeting APAF1. Nat Commun. 2016;7:11150.

83. Pink RC, Samuel P, Massa D, Caley DP, Brooks SA, Carter DR. The passenger strand miR-21-3p, plays a role in mediating cisplatin resistance in ovarian cancer cells. Gynecol Oncol. 2015;137(1):143-151.

84. Weiner-Gorzel K, Dempsey E, Milewska M, et al. Overexpression of the microRNA miR-433 promotes resistance to paclitaxel through the induction of cellular senescence in ovarian cancer cells. Cancer Med. 2015;4(5):745-758.

85. Yan X, Yin J, Yao H, Mao N, Yang Y, Pan L. Increased expression of annexin A3 is a mechanism of platinum resistance in ovarian cancer. Cancer Res. 2010;70(4):1616-1624.

86. Yin J, Yan X, Yao X, et al. Secretion of annexin A3 from ovarian cancer cells and its association with platinum resistance in ovarian cancer patients. J Cell Mol Med. 2012;16(2):337-348.

87. Zhang FF, Zhu YF, Zhao QN, et al. Microvesicles mediate transfer of P-glycoprotein to paclitaxel-sensitive A2780 human ovarian cancer cells, conferring paclitaxel-resistance. Eur J Pharmacol. 2014;738:83-90.

88. Safaei R, Larson BJ, Cheng TC, et al. Abnormal lysosomal trafficking and enhanced exosomal export of cisplatin in drug-resistant human ovarian carcinoma cells. Mol Cancer Ther. 2005;4(10):1595-1604.

89. Crow J, Atay S, Banskota S, Artale B, Schmitt S, Godwin AK. Exosomes as mediators of platinum resistance in ovarian cancer. Oncotarget. 2017;8(7):11917-11936.

90. Donnelly SR, Moss SE. Annexins in the secretory pathway. Cell Mol Life Sci. 1997;53(6):533-538.

91. Ueda K, Clark DP, Chen CJ, Roninson IB, Gottesman MM, Pastan I. The human multidrug resistance (mdr1) gene. cDNA cloning and transcription initiation. J Biol Chem. 1987;262(2):505-508.

92. Vadlapatla RK, Vadlapudi AD, Pal D, Mitra AK. Mechanisms of drug resistance in cancer chemotherapy: coordinated role and regulation of efflux transporters and metabolizing enzymes. Curr Pham Des. 2013; 19(40):7126-7140.

93. Lv MM, Zhu XY, Chen WX, et al. Exosomes mediate drug resistance transfer in MCF-7 breast cancer cells and a probable mechanism is delivery of P-glycoprotein. Tumour Biol. 2014;35(11):10773-10779.

94. Jeltsch A, Jurkowska RZ. New concepts in DNA methylation. Trends Biochem Sci. 2014;39(7):310-318.

95. Cao YL, Zhuang T, Xing BH, Li N, Li Q. Exosomal DNMT1 mediates cisplatin resistance in ovarian cancer. Cell Biochem Funct. 2017;35(6): 296-303.

96. Marchini S, Fruscio R, Clivio L, et al. Resistance to platinum-based chemotherapy is associated with epithelial to mesenchymal transition in epithelial ovarian cancer. Eur J Cancer. 2013;49(2):520-530.

97. Latifi A, Abubaker K, Castrechini N, et al. Cisplatin treatment of primary and metastatic epithelial ovarian carcinomas generates residual cells with mesenchymal stem cell-like profile. J Cell Biochem. 2011; 112(10):2850-2864
98. Kurokawa M, Ise N, Omi K, Goishi K, Higashiyama S. Cisplatin influences acquisition of resistance to molecular-targeted agents through epithelial-mesenchymal transition-like changes. Cancer Sci. 2013;104(7):904-911

99. Yew KH, Crow J, Hirst J, Pressetto Z, Godwin AK. Epimorphin-induced MET sensitizes ovarian cancer cells to platinum. PLoS One. 2013; 8(9):e72637.

100. Szajnik M, Derbis M, Lach M, et al. Exosomes in plasma of patients with ovarian carcinoma: potential biomarkers of tumor progression and response to therapy. Gynecol Obstet (Sunnyvale). 2013;Suppl 4:3.

101. Li J, Sherman-Baust CA, Tsai-Turton M, Bristow RE, Roden RB, Morin PJ. Claudin-containing exosomes in the peripheral circulation of women with ovarian cancer. BMC Cancer. 2009;9:244.

102. Baylin SB, Jones PA. Epigenetic determinants of cancer. Cold Spring Harb Perspect Biol. 2016;8(9). pii: a019505.

103. Taylor DD, Gercel-Taylor C. MicroRNA signatures of tumor-derived exosomes as diagnostic biomarkers of ovarian cancer. Gynecol Oncol. 2008;110(1):13-21.

104. Svensson KJ, Kucharzewska P, Christianson HC, et al. Hypoxia triggers a proangiogenic pathway involving cancer cell microvesicles and PAR-2-mediated heparin-binding EGF signaling in endothelial cells. Proc Natl Acad Sci U S A. 2011;108(32):13147-13152.

105. Parolini I, Federici C, Raggi C, et al. Microenvironmental $\mathrm{pH}$ is a key factor for exosome traffic in tumor cells. J Bio Chem. 2009;284(49): 34211-34222.

106. Federici C, Petrucci F, Caimi S, et al. Exosome release and low $\mathrm{pH}$ belong to a framework of resistance of human melanoma cells to cisplatin. PLoS One. 2014;9(2):e88193.

107. Escudier B, Dorval T, Chaput N, et al. Vaccination of metastatic melanoma patients with autologous dendritic cell (DC) derived-exosomes: results of the first phase I clinical trial. J Transl Med. 2005;3(1):10.

108. Zhang J, Zhang HD, Yao YF, Zhong SL, Zhao JH, Tang JH. $\beta$-Elemene reverses chemoresistance of breast cancer cells by reducing resistance transmission via exosomes. Cell Physiol Biochem. 2015;36(6):2274-2286.

109. Hu Y, Li D, Wu A, et al. TWEAK-stimulated macrophages inhibit metastasis of epithelial ovarian cancer via exosomal shuttling of microRNA. Cancer Lett. 2017;393:60-67.

110. Hu Y, Qiu LH. Study on the mechanism of macrophages inhibiting epithelial ovarian cancer cell metastasis induced by TWEAK via exosomes. Prog Obstet Gynecol. 2015;24(7):512-515. Chinese.

111. Yue Y, Wang X, Li J, Huang GR, Fan L. An experimental study on the construction of multifunctional exosome for treatment of ovarian cancer. Anti-Tumor Pharm. 2017;7(4):406-411. doi:10.3969/j. issn.2095-1264.2017.04.05.

112. Mahmoodzadeh Hosseini H, Soleimanirad J, Mehdizadeh Aghdam E, Amin M, Imani Fooladi AA. Texosome-anchored superantigen triggers apoptosis in original ovarian cancer cells. Med Oncol. 2015;32(1):409.

113. Nijman HW, van Diest PJ, Poort-Keesom RJ, et al. T cell infiltration and MHC I and II expression in the presence of tumor antigens: an immunohistochemical study in patients with serous epithelial ovarian cancer. Eur J Obstet Gynecol Reprod Biol. 2001;94(1):114-120.

114. Andre F, Schartz NE, Movassagh M, et al. Malignant effusions and immunogenic tumor-derived exosomes. Lancet. 2002;360(9329):295-305.

115. Navabi H, Croston D, Hobot J, et al. Preparation of human ovarian cancer ascites-derived exosomes for a clinical trial. Blood Cells $\mathrm{Mol}$ Dis. 2005;35(2):149-152. 
OncoTargets and Therapy

\section{Publish your work in this journal}

OncoTargets and Therapy is an international, peer-reviewed, open access journal focusing on the pathological basis of all cancers, potential targets for therapy and treatment protocols employed to improve the management of cancer patients. The journal also focuses on the impact of management programs and new therapeutic agents and protocols on

patient perspectives such as quality of life, adherence and satisfaction. The manuscript management system is completely online and includes a very quick and fair peer-review system, which is all easy to use. Visit http://www.dovepress.com/testimonials.php to read real quotes from published authors.

Submit your manuscript here: http://www.dovepress.com/oncotargets-and-therapy-journal 